\title{
Correlative 3D X-ray Fluorescence and Ptychographic Tomography of Frozen- Hydrated Green Algae
}

Yuan Hung Lo ${ }^{1,2^{*}}$, Junjing Deng ${ }^{3}$, Marcus Gallagher-Jones ${ }^{2,4}, \mathrm{Si}_{\mathrm{Chen}}^{3}$, Alan Pryor Jr. ${ }^{2}$, Qiaoling Jin ${ }^{5}$, Young Pyo Hong ${ }^{5}$, Youssef S.G. Nashed ${ }^{6}$, Stefan Vogt ${ }^{3}$, Chris Jacobsen ${ }^{3,5,7}$ and Jianwei Miao ${ }^{2}$

1. Department of Bioengineering, University of California Los Angeles, CA 90095, USA.

2. Department of Physics and Astronomy and California NanoSystems Institute, University of California Los Angeles, CA 90095, USA.

3. Advanced Photon Source, Argonne National Laboratory, Argonne, IL 60439, USA.

4. Department of Chemistry \& Biochemistry, UCLA-DOE Institute for Genomics and Proteomics, Los Angeles, California, 90095-1570, USA.

5. Department of Physics \& Astronomy, Northwestern University, Evanston, IL 60208, USA.

6. Mathematics and Computing Science Division, Argonne National Laboratory, Argonne, IL 60439, USA.

7. Chemistry of Life Processes Institute, Northwestern University, Evanston, IL 60208, USA.

* Corresponding author: lo.yuanhung@gmail.com

The ultimate goal of cellular biology is to understand the coupling between cellular structure and function in three dimensions (3D) with high resolution. The ability to do so allows life scientists to unravel how cellular morphology affects physiological properties and metabolic processes, and vice versa. Combining coherent diffractive imaging methods such as ptychography with X-ray fluorescence microscopy (XFM) can offer such correlative information [1,2]; they can provide 3D mapping of subcellular organization and elemental compositions in whole cells without sectioning or chemical labeling.

In this work [3], we performed simultaneous ptychography and XFM on an unlabeled, frozen-hydrated green alga $C$. reinhardtii and used the GENFIRE tomography algorithm [4,5] to construct high resolution 3D cellular ultrastructure. Ptychography revealed a host of electron-dense intracellular organelles while XFM highlighted their elemental content with $\sim 45 \mathrm{~nm}$ and $\sim 125 \mathrm{~nm}$ spatial resolution, respectively. This technique allowed us to examine the alga with high fidelity while avoiding cell sectioning and labeling that can compromise cell integrity and introduce undesirable artifacts.

The experiment was performed at the hard X-ray Bionanoprobe beamline [6] located in the Advanced Photon Source at the Argonne National Laboratory. The $10 \mu \mathrm{m} \mathrm{C}$. reinhardtii cell was embedded in $\sim 1.2 \mu \mathrm{m}$ thick vitreous ice on a $\mathrm{Si}_{3} \mathrm{~N}_{4}$ membrane. The sample was scanned using a focused monochromatic X-ray beam at $5.5 \mathrm{keV}$, and a silicon drift detector and pixel array detector were simultaneously triggered at each sample motion to record the fluorescence spectra and ptychographic diffraction patterns, respectively. For tomography, the sample was rotated in $2^{\circ}$ angular increments to collect a total of 63 projections ranging from $-68^{\circ}$ to $56^{\circ}$. Ptychography projections were reconstructed using a parallel implementation of the Extended Ptychographic Iterative Engine (ePIE) algorithm [7], and the ptychography phase and XFM projections were used for 3D reconstruction in GENFIRE.

Figure 1 shows reconstructed ptychography and XFM volumes of the alga, which offer several interesting observations. First, the $\mathrm{P}$ and $\mathrm{Ca}$ bodies near the bottom of the alga co-localize with the dense bodies in ptychography and are identified as ion exchanging acidocalcisomes. Second, some 
acidocalcisomes show higher $\mathrm{K}$ concentrations than cytosol, suggesting that they are in different biochemical states and could be actively exchanging $\mathrm{K}$ with the cytosol. Lastly, near the top of the alga, $\mathrm{S}$ density clearly highlights the pyrenoid, which contains S-rich RuBisCO enzymes that are involved in photosynthesis. In addition, two large faint $S$ clusters near the bottom point to possible locations of contractile vacuoles, which contain S-rich enzymes that control osmoregulation. Note that in ptychography the contractile vacuoles may be mistaken for acidocalcisomes due to their similar spherical morphology, but XFM provides extra elemental context to discern their identities.

In the past, such clarification usually required different tools such as electron microscopy and X-ray microanalysis to obtain the structure and composition. Here, however, both are acquired simultaneously. This complementary advantage, where trace elements in XFM are used to identify organelles found in ptychography, highlights the potential of correlative microscopy for studying complex biological systems. As this technique continues to advance, it will enable more sophisticated studies in areas such as nanotoxicology, biomineralization, bioenergy, and environmental remediation.

\section{References:}

[1] J Miao, T Ishikawa, IK Robinson and MM Murnane, Science 348 (2015), p. 530-535.

[2] J Deng et al., Proc. Natl. Acad. Sci. U.S.A. 112 (2015), p. 2314-2319.

[3] J Deng, YH Lo et al., Science Advance 4 (2018), p. 4548.

[4] Y Yang et al., Nature 542 (2017), p. 75-79.

[5] A Pryor et al., Scientific Reports 7 (2017), p. 10409.

[6] S Chen et al., J. Synchrotron Radiat. 21 (2014), p. 66-75.

[7] YSG Nashed et al., Opt. Express 22 (2014), p. 32082-32097.

[8] This work was supported by STROBE: A National Science Foundation Science \& Technology Center, under Grant No. DMR 1548924.

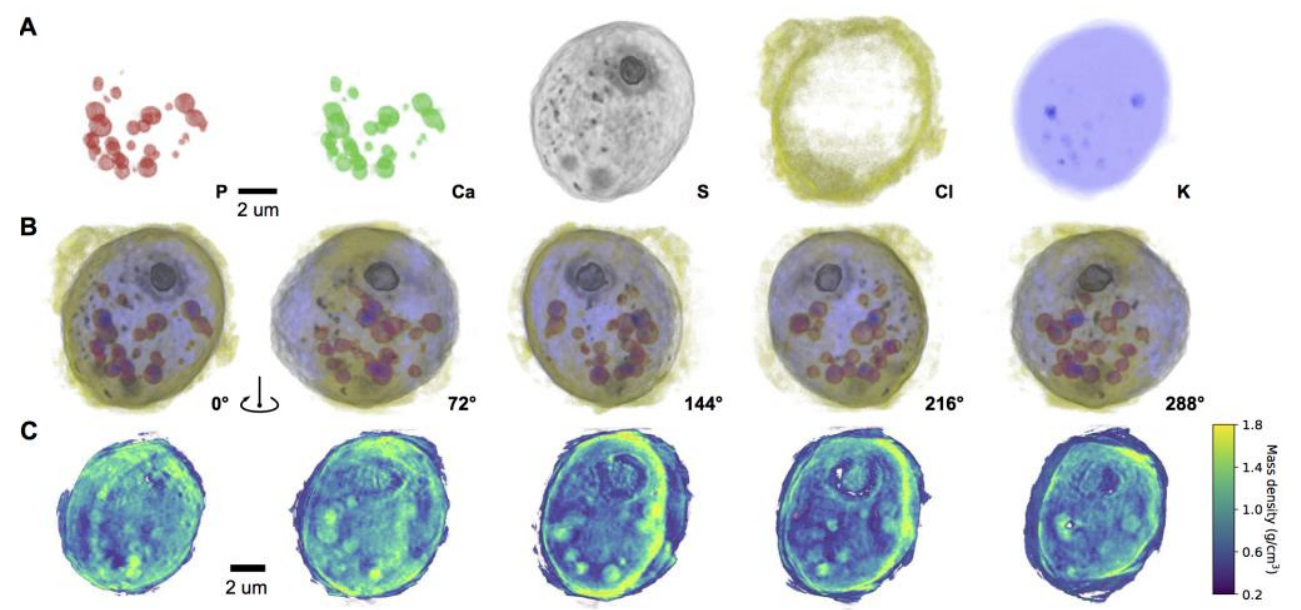

Figure 1. GENFIRE X-ray fluorescence and ptychographic tomography reconstructions. (A) $0^{\circ}$ projection of reconstructed 3D XFM volumes of $\mathrm{P}$ (red), $\mathrm{Ca}$ (green), $\mathrm{S}$ (black), $\mathrm{Cl}$ (yellow), and $\mathrm{K}$ (blue) channels. (B) Projections of the composite XFM volume, rotated in $72^{\circ}$ increments, showing localization of pyrenoid ( $\mathrm{S}$ channel) near the top and acidocalcisomes near the bottom ( $\mathrm{P}$ and $\mathrm{Ca}$ channels), along with other organelles. (C) Select $\sim 1 \mu \mathrm{m}$ slabs into the reconstructed mass density volume from ptychography, showing the pyrenoid, chloroplast and acidocalcisomes. 